\title{
Information and Communications Technologies (ICTs) for energy efficiency in buildings: review and analysis of results from EU pilot projects
}

\author{
Alberto Jáñez Morán \\ CIRCE - Research Centre for Energy Resources and Consumption (CIRCE) - CIRCE building- \\ Campus Río Ebro, Mariano Esquillor 15, 50018, Zaragoza, Spain \\ e-mail: albim@fcirce.es \\ Paolo Profaizer, María Herrando Zapater, María Andérez Valdavida and Ignacio Zabalza Bribián* \\ CIRCE - Research Centre for Energy Resources and Consumption (CIRCE) - CIRCE building- \\ Campus Río Ebro, Mariano Esquillor 15, 50018, Zaragoza, Spain \\ e-mail: pprofaizer@gmail.com, mherrando@fcirce.es, marianderez@gmail.com, izabal@unizar.es
}

*Corresponding author: izabal@unizar.es 


\section{Highlights}

- Data using two ICT systems over one year were collected for $18 \mathrm{EU}$ pilot projects

- Public buildings have achieved more than $20 \%$ energy savings due to ICT systems

- Effectiveness of the ICT solution does not depend only on the different climates

- In social housing energy savings are moderate due to the already low consumption

- Users involvement and behavioural change are crucial points for the project success

\section{ABSTRACT}

Information and Communications Technologies (ICTs) can play a potential role in improving the energy performance of buildings by the implementation of effective solutions that take advantage of the energy interactions between all the elements included in a building.

A revision of the 105 pilots implemented or under implementation in 18 projects in the area of ICTs for energy efficiency in buildings located in 23 European countries, through 88 cities with different types of climates, buildings and technologies has been carried out through documentary and field analysis of the energy, economic and social project results. These results have been extrapolated to assess the potential energy savings which could be expected at the EU level by implementing the solutions proposed by the projects.

By the implementation of the different ICT solutions, buildings have achieved more than $20 \%$ energy savings. Pilots have demonstrated that the effectiveness of the ICT solution does not depend directly on the different climates where the solutions are implemented, but on several factors, such as the level of motivation, perceived thermal comfort, quality of social interaction and communication and ICT support.

\section{KEYWORDS}

Information and Communication Technology; European Union; energy efficiency; energy savings; energy management system; public building. 


\section{INTRODUCTION}

The European Commission has recognized the potential role Information and Communications Technologies (ICTs) can play in improving the energy performance of buildings in several high-level policy documents [1-4]. ICT and ICT-based innovations may provide one of the potentially most costeffective means for Member States to achieve the 2020 target and are key to deliver the fundamental yet urgent changes required in local and regional communities.

ICT solutions for energy management and resources integration should be considered in order to help designers, stakeholders and users to increase the energy efficiency of those individual systems and the overall efficiency of the European buildings [5]. ICT solutions are very helpful to:

- Verify whether the building and the systems installed behave as designed.

- Monitor that the performance and energy efficiency are stable during the life time of the building and do not decrease.

- Achieve more energy savings combining the operation of the different systems in the building.

- Provide feedback to change user's behaviour and install new energy efficient technologies or fine tune those already installed.

- Help to increase users' comfort.

The Commission is co-financing projects and horizontal actions to support the development and deployment of energy efficient solutions for buildings under the $7^{\text {th }}$ Framework Programme for Research and the ICT Policy Support Programme (ICT PSP) which is the part of the Competitiveness and Innovation Programme (CIP) focusing on ICT, with the aim of demonstrating the energy efficiency benefits ICT can bring to building owners and their inhabitants/users.

Projects covering residential and non-residential buildings (including social housing and public buildings) have built common methodologies to calculate energy savings via ICT and the results are showing significant reductions in energy consumption and $\mathrm{CO}_{2}$ emissions of up to $20 \%$.

Two common methodologies [6, 7] for energy efficiency measurement developed by CIP social housing and public buildings projects have been designed to collect in a more harmonised way the appropriate data and produce a more meaningful quantitative analysis of the energy savings potential of ICT solutions in residential and non-residential buildings.

This analysis of results from EU pilot projects provides a comparative overview of various ICT solutions to achieve energy savings in buildings (public buildings and social housing) which were 
piloted under the CIP ICT-PSP. The objective of this study is to analyse energy usage data generated by a set of projects piloting various types of ICT systems/gadgets to reduce energy consumption in buildings (see Table 1). All projects collected the data, using the designed methodologies over a period of one year, in buildings with and without (baseline) energy saving systems/gadgets.

\section{STATE OF THE ART}

The state of the art of ICT systems for energy management at building level is discussed, focusing on the fundamental fields in which R\&D has to assume a huge challenge to start shifting the traditional and at the same time high-rigid energy management model. Five key areas including design and simulation tools, interoperability/standards, building automation, smart metering and user awareness tools have been identified [8], where there is potential to improve energy efficiency through the use of ICTs, and they are considered as the next ICT generation for future smart buildings.

According to REEB 2010 (The European Strategic Research Roadmap to ICT enabled EnergyEfficiency in Building and Construction), ICT systems have been identified as one possible means to design, optimise, regulate and control energy use within existing and future (smart) buildings. ICTs could not only be used for decreasing energy in buildings, but also to create new business opportunities driven by the need for energy efficiency. Throughout the life cycle of a building, most energy $(\sim 80 \%)$ is used during the operational stage [9]. The decisions made in the early design stages or in renovation stages for existing buildings thus influence about $80 \%$ of the total life cycle energy usage, while the impact of user behaviour and real-time control is in the range of $20 \%$ [9]. Therefore, there is an urgent need to find new possibilities to decrease the energy usage in buildings [10], for example through effective energy management of the facilities installed, such as chiller systems [11] and HVAC systems [12], especially in office buildings.

ICTs can lead to reductions on a global basis five times the size of the ICT sector's own emissions, which is $15 \%$ of total global emissions in a 'business as usual' scenario for 2020 [6]. One of the greatest roles that ICTs could play is in better design, management and automation of buildings. Industries and governments alike must find ways to utilise the possibilities.

The Building Management System (BMS) market is changing rapidly [13, 14]. Technological advances in building monitoring and control are opening the way to an era of huge volumes of data on building energy use and efficiency. Vendors are discovering innovative methods to capitalize this information to 
help buildings operate more efficiently. Although there has been a focus on energy efficiency in commercial buildings for some years, the BMS market can still be considered nascent. The landscape of new entrants, new technologies and new methodologies is expanding rapidly, and even wellestablished market leaders are finding new ways to present and promote their businesses.

\subsection{Optimisation approaches through ICTs for Energy Efficient Buildings}

Significant research efforts have been recently directed towards building energy optimisation through simulation with the objective of reducing buildings' energy consumption. For example, a recent study [15] shows a High Performance Computing (HPC) based cloud model to study and optimise the energy consumption in buildings through EnergyPlus simulation-based optimisation. To this end, the study considers several factors such as performance, cost and user perspectives, compares two different environments, determining their effectiveness to support simulation based optimisation and identifies their limiting factors through detailed performance analysis [15].

In this line, another study [16] presents a web-based parallel Genetic Algorithm (GA) optimisation framework based on distributed high-throughput computation environment (parallel NSGA-II), with which it is possible to reduce the computational time of complex buildings' energy optimization problems. This optimization framework was then tested in an experimental building in Bilbao (Spain), through its implementation in EnergyPlus, achieving a reduction in the building's energy consumption of about $15 \%$ while significantly reducing the computational time (the parallel NSGA-II was around 39 times faster than a single process GA) [16].

On the other hand, other studies, such as [17-19], focus on reducing the gap between predicted and actual energy consumption in buildings through ICTs to predict and optimise the buildings' energy consumption. In particular, Yuce B. and Rezgui Y. [17] developed an Artificial Neural Network (ANN) to learn energy consumption patterns and behaviour within the building, which can then be used as the cost function of a Genetic Algorithm (GA)-based optimization tool to generate optimized energy saving rules considering multiple objectives and constraints. This solution developed was then tested on both simulation platform and a pilot building located in the Netherlands, achieving an average of $25 \%$ energy reduction while meeting occupants' comfort conditions [17]. In this line, Yuce B. et al. [18] utilised an ANN based prediction approach to predict the energy consumption and thermal comfort 
level of an indoor swimming pool, under the EU-FP7 funded project SPORTE2 (Energy Efficient for Sport facilities [18].

Other studies, such as [19], also utilised ANN-GA for energy optimisation, in this case [19] to optimise the energy consumption in the domestic sector through an ANN-GA smart appliance controlling the energy consuming devices. This solution was then tested and demonstrated using weekly optimised schedules to achieve three levels of energy reduction, $10 \%, 25 \%$ and $40 \%$, in a four bedroom Oak House, connected to the energy grid. It was proved that the proposed approach implemented in this domestic pilot house, allowed a reduction of the energy demand in "peak" periods, maximising the use of renewable sources while reducing reliance on grid energy [19].

\subsection{Motivation of ICT systems to achieve Energy Efficient Buildings}

As shown in the previous section, a lot of efforts have been put on the development of ICTs systems to reduce the buildings' energy consumption and in general for buildings' energy management. Even though there are other methods to achieve energy efficiency in buildings, ranging from renovation/refurbishment to the use of high-efficiency appliances and adequate user behaviour, it is believed that ICT systems have a significant potential to reduce and optimise the energy consumption in buildings, as through a proper building energy management it is possible to reduce peak energy loads, switch off equipment when not in used or lighting systems when not required, etc. Furthermore, ICTs systems can be used in conjunction with other energy efficiency measures to maximise their potential, such as through automatic control of shading systems and lighting devices.

Therefore, to study and review the suitability and potential of ICTs systems in buildings, this paper presents a technology review of such technology, together with a thorough analysis of the results obtained in a series of EU pilot projects where different ICTs have been implemented in both social housing and public buildings. The 105 pilots considered are located in different types of climates and building types and used diverse technologies, covering a broad and heterogeneous sample from which the results have then been extrapolated to assess the potential energy savings that could be achieved at EU level thanks to the implementation of the ICT solutions proposed within the project. To complement this study, an economic and social analysis have also been undertaken to have a broaden and more in-depth point of view of the cost associated to these systems as well as their 
interaction with people, which are the limitations that hinder their use and how to overcome them. Finally, a replication plan of the ICT solutions is also presented.

\section{TECHNOLOGY REVIEW}

The ICT infrastructure design consists on the selection and integration of the following items (see Figure 1):

- Monitoring devices: these will depend on the systems in the building and the parameters to be measured.

- Communication infrastructure: it is required to transmit the measurements from the monitoring equipment to the control centre, and the orders from the control centre to the field actuators.

- BMS: it is the software collecting field measurements and displaying them to the users. It can also control remotely and automatically the different systems in the building based on different algorithms.

It is believed that the main challenge in the design of the ICT infrastructure is the vast number of possibilities available for the previous items, most of them very difficult to integrate in a single comprehensive ICT management system for the building. A large variety of different energy management products in buildings from big corporations to Small and Medium-Sized Enterprises (SMEs) compete in the market, which raise several doubts and concerns among potential customers, hindering the decision-making process because of lack of confidence on the suitability and usefulness of the most appealing options. This leads to the loss of many opportunities to improve energy efficiency in buildings.

This problem becomes even more important in existing buildings, with several stakeholders taking part during the building life cycle, many of them small SMEs using their own technology depending on their know-how or expertise, and usually, no one leading an overall strategy.

Retrofitting of buildings can be a good opportunity to install some of the BMS features. If the implementation of an active controlling system is not possible, at least measuring devices for the visualization of energy use should be considered to promote user awareness. Systems with wireless communication and even self-powering might be a good alternative for existing buildings where installation works cannot be performed in full scale. Also, the project-oriented approach of building industry makes the adoption of long-term strategies very complex. That is why it is very important to 
adopt a long term ICT energy efficiency policy whenever possible from the early stages in the building design.

Within this context, the most common components of the ICT systems are defined in the following subsections.

\subsection{Sensors and Smart Metering}

Energy optimisation of buildings requires the possibility to analyse the characteristics and performance of buildings [20]. ICT technologies can provide an ideal solution to perform the monitoring of several physical and energy consumption parameters. According to these parameters, sensors can be divided in two main categories:

- Sensor for monitoring physic parameters: indoor and outdoor temperature, humidity, lighting, human occupancy, aperture, $\mathrm{CO}_{2}$ concentration, etc.

- $\quad$ Sensor for monitoring consumption: gas, electricity, water, etc.

The role of the sensors within an energy management system is to provide an environmental context in which users and automated mechanisms can validate the mode of operation and take corrective actions if necessary (see Table 2), for example by adjusting the heating system according to a thermostat or turning off lights when there is no occupancy [21].

\subsection{Communication protocol}

Communication between sensors, actuators and BMS is of particular importance to ensure the proper control and management of the building. A network is not an energy management application, but it is the glue that enables energy management applications to monitor and control the devices. Existing protocols are either owned by private technology providers or open communication languages.

Thanks to the communication technologies, sensors make the energy context and other physical parameters visible, and then building management systems can monitor and optimise the operation, enabling appliances to obtain enough information to consume energy responsibly on their own by using real-time data loaded from power distribution systems; and also providing feedback of their realtime status and energy consumption.

Besides this, in the future, the connection with power distribution systems will be feasible, so energy management applications will be required to optimally operate the power sources available, adjust the 
mode of operation or improve the peak load management responding to grid events. Again, standards, protocols and communication technologies are the ones which will make this plausible.

The different technologies can be classified based on the transmission medium into wired and wireless $[22,23]$. The reason of this division is that their requirements are different. For example, power consumption is a very important factor in wireless technologies [24], but far less important in wired.

On the other hand, the speed of the technologies depends mainly on the physical layer for wireless technologies [25], and on the physical medium and the codification for wired, so the technologies that use the same means of transmission will have similar speed and range. There are multiple configuration possibilities for each protocol and new versions are released improving what the former was not able to achieve [26].

The most usual protocols for the building sector among Europe are [27]: BACnet (open) [28], KNX (open) [29] and LonWorks (open).

\subsection{Building Management System (BMS)}

Fully equipped buildings need control and management systems in order to coordinate at least the various active systems working to produce heat, cold and clean air and their relation with the variable weather predictions [30]. Building management is also becoming compulsory for new buildings with low energy consumptions in order to ensure not only the correct performance of the systems but also their effectiveness. Nowadays, BMS are implemented systematically in tertiary buildings [31] and have an increasing implementation in the residential sector.

BMS is a computer-based control system installed in buildings that controls and monitors the building's mechanical and electrical equipment such as Heating, Ventilation and Air Conditioning (HVAC), Closed-Circuit Television (CCTV), Access Control, Fire and Intruder Alarms, Lighting and Power systems (see Table 3 for more details about the BMS skills). The EN 15232 is the standard concerning the energy impact of building automation, control and building management. It also provides a good overview of common off-the-shelf control functionalities in a BMS.

BMS complexity ranges from covering some of the next four basic functions to include all of them, that is, Controlling, Monitoring, Optimising and Reporting. Nowadays, monitoring and reporting are common features for BMS, even in small projects for housing. However, BMS covering the four functions are more commonly implemented in large projects for tertiary service buildings with 
extensive mechanical, electrical, and pumping systems. Systems linked to a BMS typically represent $40 \%$ of a building's energy usage; if lighting is included, this number approaches $70 \%$ depending on the type of building.

With a centralized BMS reporting on high yield energy consumption facilities, power consuming systems are easily identified. These reports help in building automation fine tuning to save energy, and encourage initiatives such as cost justification to invest in more energy efficient systems whilst simultaneously promoting the awareness of energy efficiency throughout the organization.

Focusing on how automation can help energy efficiency in BMS with control functionalities, there are numerous ways in which building services can be controlled, with three basic methods:

- Time based: installing a timer to provide such services for instance for lighting and HVAC systems, when required.

- Occupancy based control: the use of presence detectors to control lighting and indoor conditions, as well as the business schedule.

- Parameter optimisation based: through the optimisation of a representative aspect, such as temperature for space heating.

For many years now, there are independent systems (HVAC, lighting, microgeneration and power distribution, etc.) in buildings which use the previous control methods considering them as stand-alone measurements of energy efficiency, but further energy reductions not commonly exploited are possible through, for example, combining heating, lighting and blind-control. However, these combined solutions are only available in the most advanced commercial BMS or in tailored BMS designed with commercial Supervisory Control and Data Acquisition (SCADA) tools.

Although it is still far from being common, some emerging applications of predictive control modelling are also applied to buildings. In this case, a conventional BMS manages the building, but at the basis of this system there is an optimisation software tool that operates the BMS, e.g. changing the temperature set-point within a comfort interval taking into account information such as the weather forecast, real-time energy pricing, etc., to achieve an optimal trade-off between energy consumption, comfort and economy. 


\section{DATA ANALYSIS FROM EU PILOT PROJECTS}

\subsection{Projects' buildings details}

The main representative details of the different buildings used as pilots for the ICT systems' implementation is shown in this section.

\section{Number of buildings and number of users involved}

As shown in Table 4, the social housing and public buildings projects considered have a significant impact on the community where they are developed involving a significant number of buildings and citizens.

\section{$\underline{\text { Buildings location by climatic region }}$}

Social housing and public buildings projects have implemented and developed their pilots across Europe covering the main European climatic regions and countries as it is shown in Figure 2.

\section{Buildings distribution by year of construction}

As it is shown in Figure 3, it is clear that the ICT solutions implemented by the projects are suitable not only for new or recent buildings but also and especially for old buildings both in social housing and public buildings.

\section{Typologies of public buildings}

The public buildings projects have implemented ICT solutions in different typologies of buildings in order to prove that their use can help building users to reduce the energy consumption adapting these solutions to the peculiarities of the various activities that take place within public buildings (see Figure $4)$.

\subsection{Actions piloted by the projects}

On one hand, there are two main system typologies:

- Energy Awareness Systems (EAS): These systems deliver to users real-time data on their current energy consumption at their house or at a public area in order to make them able to save energy by adapting their energy consumption behaviour. Behaviour transformation as a concept is based on the use of empirically demonstrated techniques as a means to impact on the behaviour of individuals, and thus to achieve an objective identified. Real-time data is an added value providing that the adequate message is passed on to users. By comparing the 
real-time data to the baseline, users can perceive energy consumption reductions or increases compared to a business-as-usual profile.

- Energy Management Systems (EMS): These systems deliver information about general parameters measurements such as temperature, humidity and consumptions, as well as the main technical parameters of the equipment. By measuring these parameters, the system can set specific alarms and a synthesis of the building's performance both at real-time and for the past periods, allowing an interesting analysis of the energy and comfort parameters of the building. EMS helps building managers to administer their buildings better, enabling more efficient energy management, fairer energy billing, the introduction of more powerful incentives for energy savings, and intelligent electricity peak savings. These systems can also incorporate a control device used for the whole control and maintenance of buildings services, allowing the management of all the HVAC equipment and lighting systems in relation to exterior and interior conditions, occupancy and pre-programmed scenarios. The building's operation and management staff should know how to use the monitoring system installed in the building in order to reduce the energy consumption on a daily basis through a continuous monitoring.

On the other hand, the system users are identified in two main groups, according to their position regarding the technological solutions. These users groups need different ICT tools to contribute to the energy savings of the building:

- Technicians: They are dealing directly with ICT solutions and can influence them. They work to reduce the energy consumption of the building by using EMS such as:

- Communication infrastructure: real-time measurements of the energy production and consumption.

- Specific software tools: for overall system management and general integration of the monitoring systems, giving the complete EMS an added value.

- Users of the building: They are dealing indirectly with ICT solutions and are affected by them. They can influence in the reduction of the energy consumption of the building by the use of EAS such as:

- Public information systems: showing real-time information on the energy consumption and the energy efficiency. 
- Messages: awareness rising to ask for some kind of action.

- Control about own behaviour and comfort: regulation of lighting, heating, cooling, water and electricity use.

The technical actions piloted by projects for the monitoring and control of the energy consumption in social housing through the implementation of ICT are divided in two main levels: dwelling level and building level, due to the different type of information managed by the two main services implemented in the projects, EAS (related to tenants) and EMS (related to technicians or building managers). For public buildings, only building level is considered.

Figures 5 and 6 show the distribution of the different types of energy savings actions applied to social housing and public buildings respectively. Regarding social housing projects, Figure 5 shows that energy saving actions in electricity are the most predominant, both at dwelling and building level, with $82 \%$ and $55 \%$ of the pilots respectively. It is also possible to observe that, even though both levels show the same trends, at dwelling level substantially more actions were taken in Heating and Domestic Hot Water (DHW), as expected. Similarly, Figure 6 shows that, in the case of public building projects, $96 \%$ of the pilots implemented energy savings actions in electricity, while only $14 \%$ implemented Renewable energy technologies.

\section{RESULTS AND DISCUSSION}

This section shows a discussion of the main results of the pilot projects regarding the energy savings achieved, an economic and social analysis, as well as the potential of replication at European level.

\subsection{Energy savings analysis}

The analysis of social housing pilots in different countries achieved promising results, showing that the use of ICT solutions in households is a very useful tool to save energy. An in depth analysis of the results indicated that, even though almost $50 \%$ of the pilot sites achieved the $20 \%$ energy savings target defined in the evaluation plan, in several cases it is difficult to reach this target due to the following reasons:

- Electricity consumption is becoming progressively more difficult to reduce as people increasingly have more electrical devices (tablets, PCs, smartphones, etc.). 
- The average energy consumption of the social housing pilot dwellings is already significantly lower than the average energy consumption of each country.

- ICT systems should be properly explained to the end users. Furthermore, the better relationships with the tenants, the better the results are.

- The energy efficiency and $\mathrm{CO}_{2}$ reductions achieved thanks to the implementation of ICT systems depend on the specific training of professionals and users of these technologies, which implies a learning curve resulting in potentially higher energy savings in the near future.

Figure 7 shows a summary of the energy savings achieved in the social housing pilots analysed.

First of all, one should bear in mind that heating is the main energy consumption in residential buildings, therefore there is an important opportunity of achieving savings in heating and Domestic Hot Water (DHW) through the optimisation of the central heating system, and in particular improving its operation. It is estimated that the energy savings potential through optimisation of the operation ranges from $10 \%$ to $20 \%$ depending on the real state of the heating system. In addition, it is believed that with an intensive use of the EAS, which shows important information about energy consumption and costs, tenants could progressively change their behaviour, reducing their energy consumption by approximately $8 \%$ on average. In the case of public buildings, the results show that it is more efficient to concentrate first the efforts on optimising the technical system. However, it was observed that the pilots achieved significant energy reductions not only through this optimisation but also thanks to the improvement in operational procedures, the increase of user's involvement and the enhancement of the interactions between users, technologies and buildings.

Of the total energy savings that the pilots achieved, half of them were because of the investments in building services retrofit (mainly in HVAC and lighting systems) and the other half due to the impact of the ICT system implemented. Figure 8 shows a summary of the energy savings achieved within the public building pilots analysed. It is possible to observe that in this case most of the buildings achieved the $20 \%$ energy savings target established, with some pilots achieving more than $50 \%$ energy savings (HosPilot project in Spain and France and EDISON project in Belgium).

\subsection{Potential energy savings at European level}

The European social housing stock is estimated in $9.5 \%$ of the total European dwelling stock with a total area of $1,781.25$ million $\mathrm{m}^{2}$. The potential savings regarding energy, $\mathrm{CO}_{2}$ emissions and 
economics for the social housing sector at European level have been extrapolated from the results obtained in the EU projects studied in the present work and are shown in Table 5.

On the other hand, non-residential buildings account for $25 \%$ of the total building stock in Europe with a total area of 1,214.9 million $\mathrm{m}^{2}$ and comprise a more complex and heterogeneous sector compared to the residential sector. The retail and wholesale buildings make up the largest portion of the nonresidential stock while office buildings are the second biggest category with a floor space corresponding to one quarter of the total non-residential floor space. The total non-residential floor space within the EU is divided, on average, as follows: $28 \%$ wholesale and retail, $23 \%$ offices, $17 \%$ education, $11 \%$ hotels and restaurants, $7 \%$ hospitals, $4 \%$ sport facilities and $11 \%$ others. Considering these numbers, Table 6 shows the potential savings regarding energy, $\mathrm{CO}_{2}$ emissions and economics for the public buildings at European level.

\subsection{Economic analysis}

First of all, it should be noted that the ICT system costs analysed are higher than expected. In social housing, the installation costs ranges from $300 €$ to $1,000 €$ per dwelling, depending on the number of installations monitored and the average country prices. The payback period depends on the average energy cost; therefore in some countries the current energy prices are too low to calculate a positive Return Of Investment (ROI). Nevertheless, the financial pay-off is achieved by most social housing tenants during the first 5 years and by almost all after 10 years.

On the other hand, in tertiary buildings, the average ICT system installation cost is approximately $6.25 € / \mathrm{m}^{2}$. These high costs may be due to the unfamiliarity that the installers still have with the requirements. However, the overall solution costs are likely to decrease over the next years, once standards are established. It should be considered that the ROI depends also on the use of the buildings, as some buildings are higher energy consumers than others. All the results obtained infer that, economically, it is more efficient to concentrate the first efforts on EMS than on EAS, and to focus first on buildings and services that are more energy consumers. After the optimisation on EMS, the generated savings can be used to invest in EAS and systems based on direct feedback, which have a longer ROI due to the need of users to adapt their behaviour.

It can be concluded that, in general, for the implementation of ICT systems, both in social housing and public buildings, it is necessary the development of economies of scale and cost reduction of equipment. 


\subsection{Social analysis}

From the social point of view, the effectiveness of the ICT solution depends on several factors, such as the level of motivation, perceived comfort, quality of social interaction and communication and ICT support. Besides, one should bear in mind that the way of thinking, feeling and acting of end-users are not only influenced by external factors, but also by internal aspects like personal habits, values, experiences and interests on the project.

In the case of social housing, the involvement of the tenants and their behavioural change is a crucial point for the success of the ICT project that requires an intrusion and an installation of a new technology and devices in a private home. The lack of motivation is usually related to a low education in energy issues and by the fact that social housing tenants have already low energy consumption because of economic difficulties. To overcome these difficulties, the projects analysed empower consumers with a greater understanding of their energy costs and tariffs, making them able to switch their energy supplier to receive a cheaper tariff, which could reduce the extent and effects of fuel poverty.

All the activities which guide to increase the awareness of the tenants should be adapted to the different typology of users and their level of technological knowledge. As an example, the use of a tablet is easier for young users, but it can be extremely difficult for elderly people without a digital background. Due to this difference between users, it is crucial to select different awareness intervention strategies for each specific target group. A participatory collective training session or workshop is a very effective strategy to develop and raise awareness of the target group while, on the other hand, it gives the target group the possibility to get involved and engaged in the project.

In the implementation of the ICT system in households, locally active energy managers and social workers are essential to explain how the services operate and the advantages of using them, as face to face information is generally more effective than brochures.

In public buildings, there are many people involved in the implementation of an ICT solution, such as visitors, staff, technicians, building managers and users, which imply to need to implement different actions for different typology of users in order to increase awareness of energy efficiency by changing user's behaviour.

Motivation and education of building users can have a significant impact on energy efficiency in a building, even if permanent energy savings are often achieved with technical measures. To his end, it 
is important to use a range of user behaviour transformation tools. For instance, a real time energy data display with no additional user behaviour transformation tools has a minimal impact on its own. Consequently, a strategic involvement plan should include informal meetings, real time displays with information on energy consumptions, workshops, weekly or daily emails including energy savings information and energy savings suggestions and reminders, interactive digital games and use of social media in order to maintain involved in the project all the affected users.

Furthermore. in order to increase the motivation of people, maximise personal contact is one of the keys for the success of the project and also to achieve the goal of behavioural change in addition to interact and integrate with other energy savings initiatives in the same city or country or with the same typology of buildings. The involvement plan may also influence the relatives of the building users. For example, if applied to a school, the pupils involved, in addition to play with interactive learning games within the classes, they can spread the awareness of energy efficiency to their families when they arrive home.

Finally, another conclusion extracted from the results is that it is not only important to focus on the staff and the employees, behavioural change should also be promoted on all relevant levels such as the higher management, the service personnel, the guards, the building management and the installation companies. Individuals need to see the organization acting in the same way that they are being asked to behave; otherwise, they will lose interest in transforming their behaviour.

\subsection{Replication plan of the ICT solutions}

The main steps of a replication plan are shown in Table 7 . The first steps are the definition of the objectives, boundary conditions and project limitations. At this stage, it is useful to evaluate the existing policies and goals related to the energy efficiency in buildings, at international, national and local level, and also evaluate the users and their needs to achieve the goals.

Afterwards, an energy efficiency audit is convenient for an initial check of the energy savings potential of the building. A technical audit gives an overview of the energy operation of the building which will help to make decisions to improve the installations. Moreover, the basis of any suggestion of any measure to reduce energy consumption need detailed measured consumption data. The availability of high quality data to calculate the baseline consumption is very important to ensure the reliability of the results. Based on an energy audit and on the needs of the users, the defined goals and the technology 
chosen can be analysed also by their costs and ROI in order to measure the efficiency and the sustainability of the investment.

It is strongly recommended that ISO 50001 is used as the basis for energy savings programs. To measure the change in energy consumption requires a robust process and, in the case of buildings, this process should be based on the International Performance Measurement and Verification Protocol (IPMVP). In this line, an independent energy management certification, like ISO 50001, would be a helpful source to develop the corresponding rules in buildings. Besides, regulations such as ISO 50001 focus on the physical implementation, management and operation of energy savings equipment, which is the key to improve the energy efficiency of buildings. The continuation of the whole energy management process by a certification organization from the beginning of the design, over the implementation of the technology, to the assessment of the results would ensure a reduction of technical and user related dysfunctions of the entire energy management process.

After the selection and implementation of the ICT system and the user engagement, and once the analysis and optimisation of buildings performance is carried out, the next step is the monitoring and analysis of the data collected and the results achieved that include correction and comparison of the data. The Measurement and Verification (M\&V) methodology is intended to promote good practices and consistency in the reporting of any project results. The use of the methodology should also assist others to identify more clearly potential energy savings opportunities including the development of local and national policy. In summary, a M\&V plan mainly focuses on meter installation, calibration and maintenance, data gathering and screening, development of computation methods and, if necessary, acceptable estimates, computation of measured data and reporting, quality assurance and third-partyverification of reports. In this line, it is also important to calculate the energy indicators, to analyse and describe significant deviations precisely and to compare the targeted and achieved results.

The last step is the dissemination of the results including a clear and transparent interpretation and communication of the project results to the owners and the top management as a recommended step to achieve a successful continuity of the energy efficiency program.

\section{CONCLUSIONS}

While complex systems are required in tertiary buildings, houses are usually equipped by localised systems adapted to one dwelling with simplified information and management possibilities. Customers 
can follow real time energy consumption in specific areas, and adapt their behaviour accordingly. In households, energy savings generated by ICT systems are moderate to low, compared to the impacts generated by envelope improvement or modification of HVAC systems in the buildings. This leads to a modest, but very interesting level of savings generated by behavioural changes only.

Domestic ICT systems send alerts and guidelines for energy savings to tenants' electrical devices such as their computer, television or tablet; or alternatively a set of personalized recommendations can be presented on paper by mail post or in a personal meeting with an energy coach. This the information provided, end-users are also able to compare their consumption to the corresponding one of other tenants or to the 'average' tenant. Within the information provided, historical values are available so as the tenants can observe their energy consumption over the years, which will allow them taking appropriate actions in case they consider their energy consumption should be lowered. Besides, some of the EAS create tips for tenants on how energy consumption and costs can be saved based on the observed consumption patterns and figures.

It should be kept in mind that users' comfort is not only an important issue in private households. In public buildings it has at least the same importance or even more than in private houses, as it is believed that employees working in public office buildings perceive their personal space/office climate as the most important issue within their jobs.

It is has found that the biggest barrier in the implementation of energy efficiency measures in public buildings is that there are many people involved in energy management and usually the people involved in the energy consumption are not the same as those who benefit financially from energy savings. This financial incentive gap is hence a key factor in the selection of the user's behaviour transformation tools.

Anyhow, it is believed that the successful results that can be achieved in energy efficiency due to user behaviour transformation supported by real time information in public buildings make essential for municipalities to invest in the implementation of ICT systems in such pilots, and to become rolemodels of energy efficiency in their communities, leading the way for the replication of the same strategy by private stakeholders in service buildings, and influencing citizens to apply similar behaviour in their homes/industries and through networks of cities. 


\section{NOMENCLATURE}

ANN - Artificial Neural Network

BMS - Building Management System

CCTV - Closed-Circuit Television

DHW - Domestic Hot Water

EAS - Energy Awareness Services

EMS - Energy Management Services

GA - Genetic Algorithm

HPC - High Performance Computing

HVAC - Heating, Ventilation and Air Conditioning

ICT - Information and Communication Technology

IPMVP - International performance measurement and verification protocol

REEB - The European Strategic Research Roadmap to ICT enabled Energy-Efficiency in Building and Construction

RMS - Resource Management Services

ROI - Return Of Investment

RUAS - Resource Use Awareness Services

SCADA - Supervisory Control and Data Acquisition

SEP - Smart Energy Platform

SME - Small and Medium-Sized Enterprise

SSL - Solid State Lamps

STP - Sewage Treatment Plan

TCP/IP - Transmission Control Protocol and the Internet Protocol

VLC/PLC - Visible Light Communications and Powerline Communications

WTP - Water Treatment Plan

\section{ACKNOWLEDGENTS}

This paper was developed from the results obtained in the framework of the study "SMART 2013/0073 - Reducing energy consumption in buildings with ICT - analysis of data from EU pilot projects" 
financed by the European Commission - DG Communications Networks, Content \& Technology and executed by CIRCE.

The sole responsibility for the content of this publication lies with the authors. It does not necessarily reflect the opinion of the European Union. Neither the EACl nor the European Commission are responsible for any use that may be made of the information contained therein.

\section{REFERENCES}

1. COMMUNITIES, C.O.T.E., Communication from the Commission to the European Parliament, the Council, the European Economic and Social Committee and the Committee of the Regions on mobilising Information and Communication Technologies to facilitate the transition to an energyefficient, low-carbon economy. 2009.

2. COMMISSION, E., Commission recommendation of 10 October 2014 on the Data Protection Impact Assessment Template for Smart Grid and Smart Metering Systems (2014/724/EU). Official Journal of the European Union, 2014.

3. Group, H.-L.A. and R. Consortium, ICT for a low carbon economy. Smart buildings. European Commission Directorate-General information society and media, 2009.

4. COMMISSION, E., Communication from the Commission to the European Parliament, the Council, the European Economic and Social Committee and the Committee of the Regions. A digital agenda for Europe. 2010.

5. Kramers, A., et al., Smart sustainable cities - Exploring ICT solutions for reduced energy use in cities. Environmental Modelling \& Software, 2014. 56(0): p. 52-62.

6. Webb, M., SMART 2020: Enabling the low carbon economy in the information age. Global eSustainability Initiative (GeSI), 2008.

7. Woodall, G., D1..2 Non-residential methodology. SMART 2011/0072: Methodology for energyefficiency measurements applicable to ICT in buildings (eeMeasure), 2011.

8. Ye, J., T.M. Hassan, and C.D. Carter, ICT for Energy Efficiency: The Case for Smart Buildings. Department of Civil and Building Engineering, Loughborough University.

9. Hannus, M., A. Samad, and A. Zarli, ICT supported energy efficiency in construction - Strategic research roadmap and implementation recommendations. REEB - European strategic research roadmap to ICT enabled energy-efficiency in buidlings \& construction, 2010.

10. Kramers, A. and O. Svane, ICT applications for energy efficiency in buildings. Report from the KTH Centre for Sustainable Communications, 2011.

11. Wei, X., G. Xu, and A. Kusiak, Modeling and optimization of a chiller plant. Energy, 2014. 73: p. 898-907.

12. Tang, F., A. Kusiak, and X. Wei, Modeling and short-term prediction of HVAC system with a clustering algorithm. Energy and Buildings, 2014. 82: p. 310-321.

13. Bloom, E. and R. Martin, Building energy management systems: overview and forecast. GIGAOM Research, 2013.

14. Ishida, $\mathrm{H}$., The effect of ICT development on economic growth and energy consumption in Japan. Telematics and Informatics, 2015. 32(1): p. 79-88.

15. Petri I, L.H., Rezgui Y, Yang C, Yuce B, Jayan B, , A HPC based cloud model for real-time energy optimisation. Enterprise Information Systems, 2014.

16. Yang, C., et al., High throughput computing based distributed genetic algorithm for building energy consumption optimization. Energy and Buildings, 2014. 76: p. 92-101.

17. Yuce B; Rezgui Y, An ANN-GA semantic rule-based system to reduce the gap between predicted and actual energy consumption in buildings. IEEE Transactions on Automation Science and Engineering, 2015: p. 1-13

18. Yuce, B., et al., Utilizing artificial neural network to predict energy consumption and thermal comfort level: An indoor swimming pool case study. Energy and Buildings, 2014. 80: p. 45-56.

19. Yuce, B., Y. Rezgui, and M. Mourshed, ANN-GA smart appliance scheduling for optimised energy management in the domestic sector. Energy and Buildings, 2016. 111: p. 311-325.

20. Hajdukiewicz, M., et al., Real-time monitoring framework to investigate the environmental and structural performance of buildings. Building and Environment, 2015. 86: p. 1-16. 
21. Hardy, A., M. Merabti, and F. Bouhafs, (IJAEST) INTERNATIONAL JOURNAL OF ADVANCED ENGINEERING SCIENCES AND TECHNOLOGIES, 2011. 3(2): p. 061-077.

22. Sriskanthan, N., F. Tan, and A. Karande, Bluetooth based home automation system. Microprocessors and Microsystems, 2002. 26(6): p. 281-289.

23. Gravogl, K., J. Haase, and C. Grimm, Choosing the best wireless protocol for typical applications. Institute of Computer Technology, Vienna University of Technology, 2011.

24. Marsic, V., et al., Design and implementation of a wireless sensor communication system with low power consumption for energy harvesting technology. 19th Telecommunications Forum (TELEFOR), 2011.

25. Egan, J., Multiple Input, Multiple Output Technology Accelerates Wired Home Networks. HomeGrid Forum white paper, 2010.

26. Kailas, A., V. Cecchi, and A. Mukherjee, A Survey of Communications and Networking Technologies forEnergy Management in Buildings and Home Automation. Journal of Computer Networks and Communications Hindawi Publishing Corporation, 2011. 2012.

27. Gee Keng, E., et al., A Review of 6LoWPAN Routing Protocols. Proceedings of the Asia-Pacific Advanced Network, 2010. 30: p. 71-81.

28. Pang, X., et al., A framework for simulation-based real-time whole building performance assessment. Building and Environment, 2012. 54: p. 100-108.

29. Nývlt, O., Buses, Protocols and Systems for Home and Building Automation. Evropský sociální fond. Praha \& EU: Investujeme do vaší budoucnosti, 2011.

30. Lazos, D., A.B. Sproul, and M. Kay, Development of hybrid numerical and statistical short term horizon weather prediction models for building energy management optimisation. Building and Environment, 2015. 90: p. 82-95.

31. Kyselytsya, Y. and T. Weinzierl, Implementation of the KNX Standard. WEINZIERL ENGINEERING GmbH, 2006. 


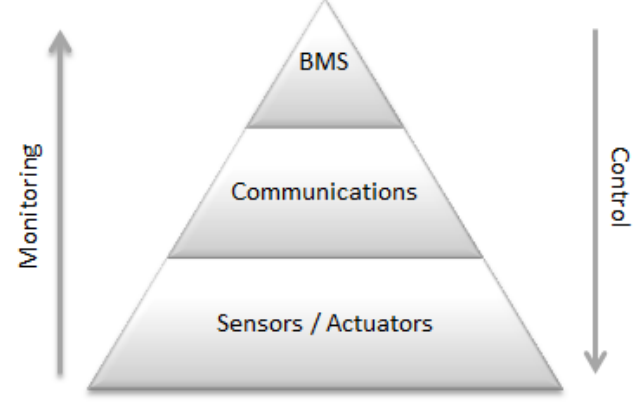

Figure 1. ICT infrastructure 


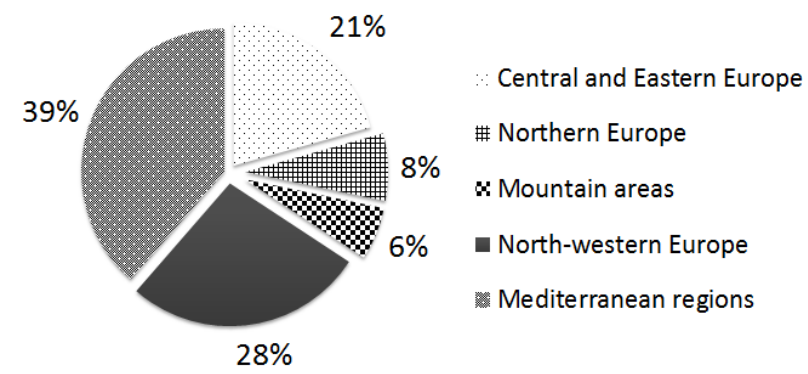

Figure 2. Buildings location by climatic region. 


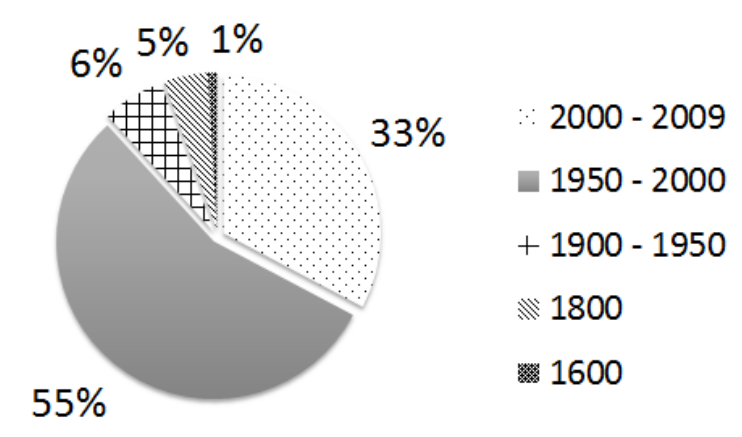

Figure 3. Buildings distribution by year of construction. 


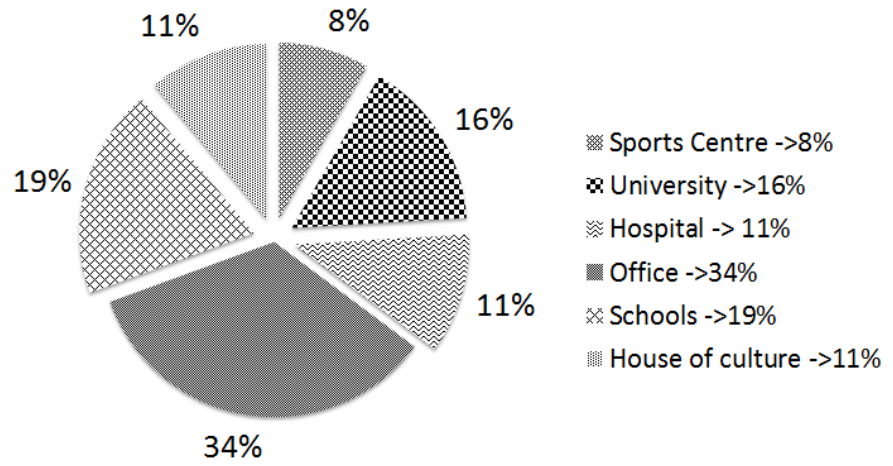

Figure 4. Public buildings distribution by typology. 


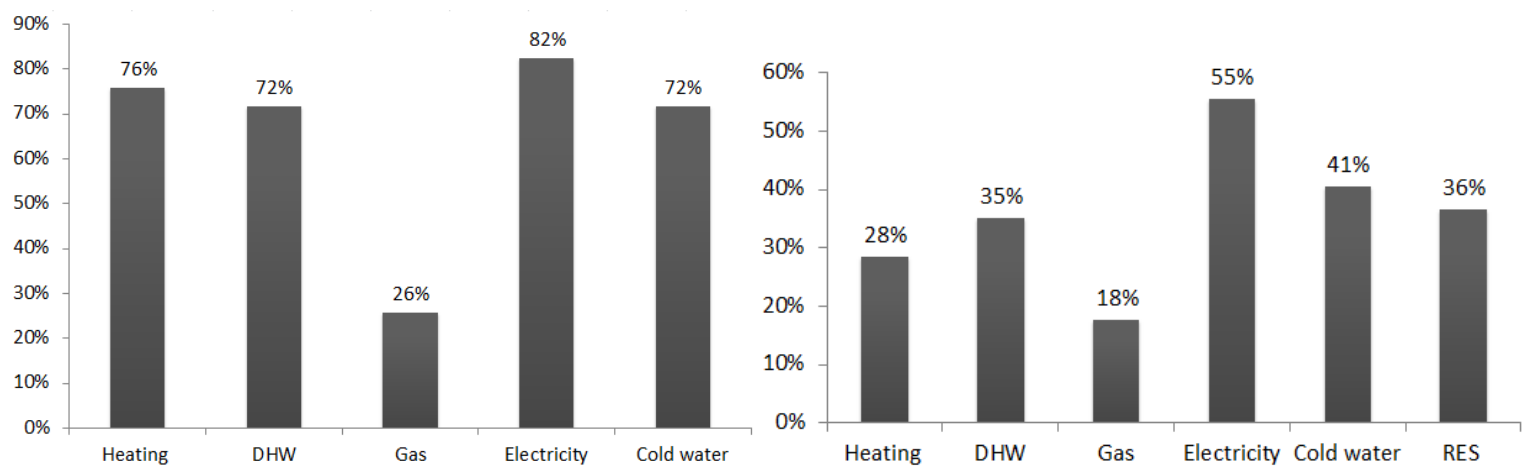

Figure 5. Energy savings piloted by social housing projects at dwelling level (left) and building level (right). 


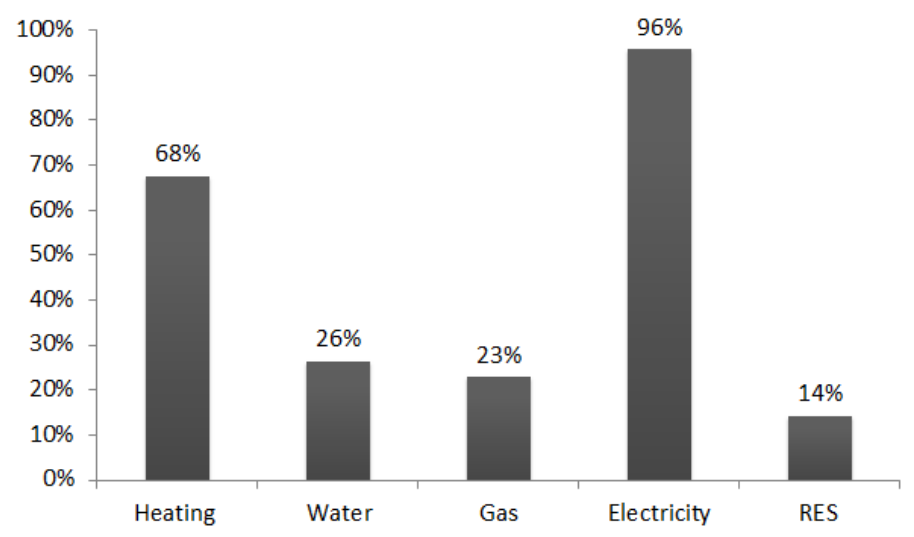

Figure 6. Energy savings piloted by public buildings projects. 


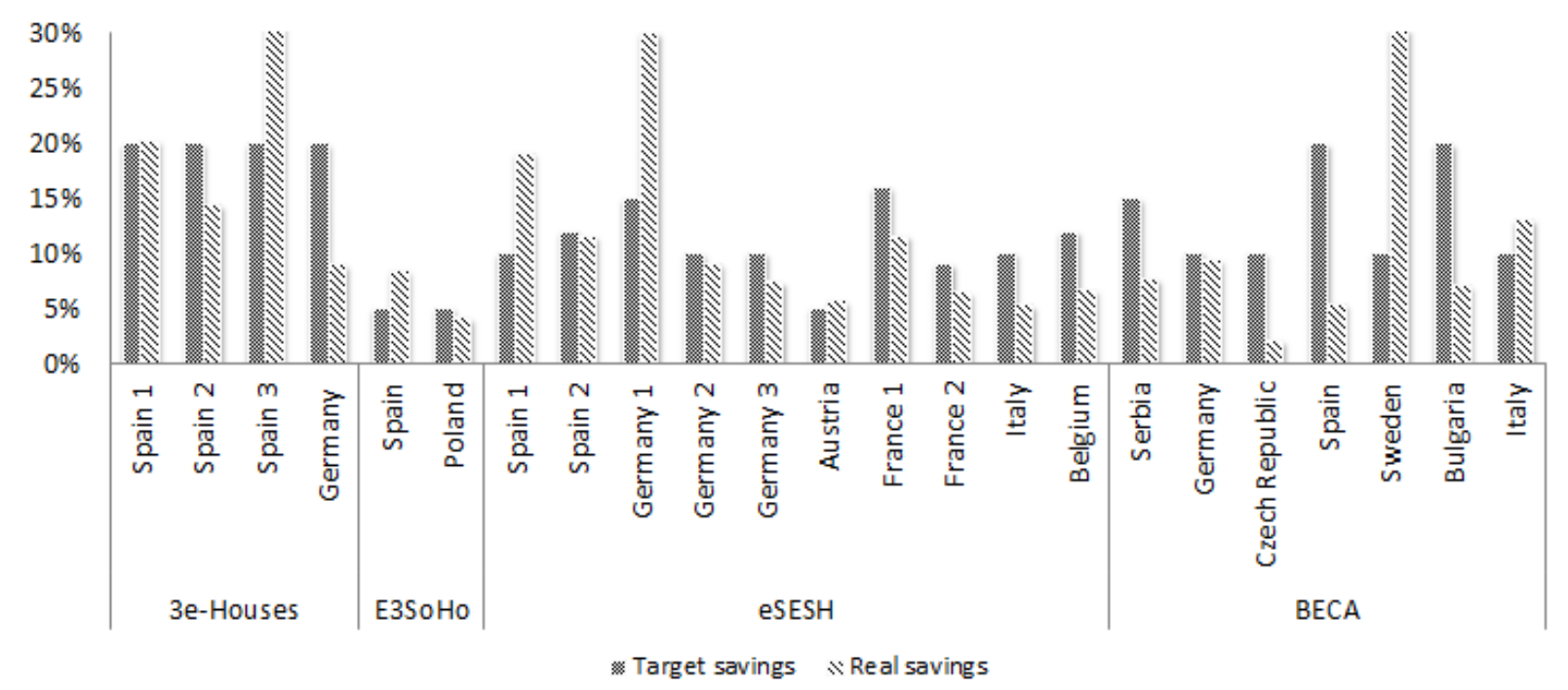

Figure 7. Total percentage of energy savings achieved within the different social housing pilots. 


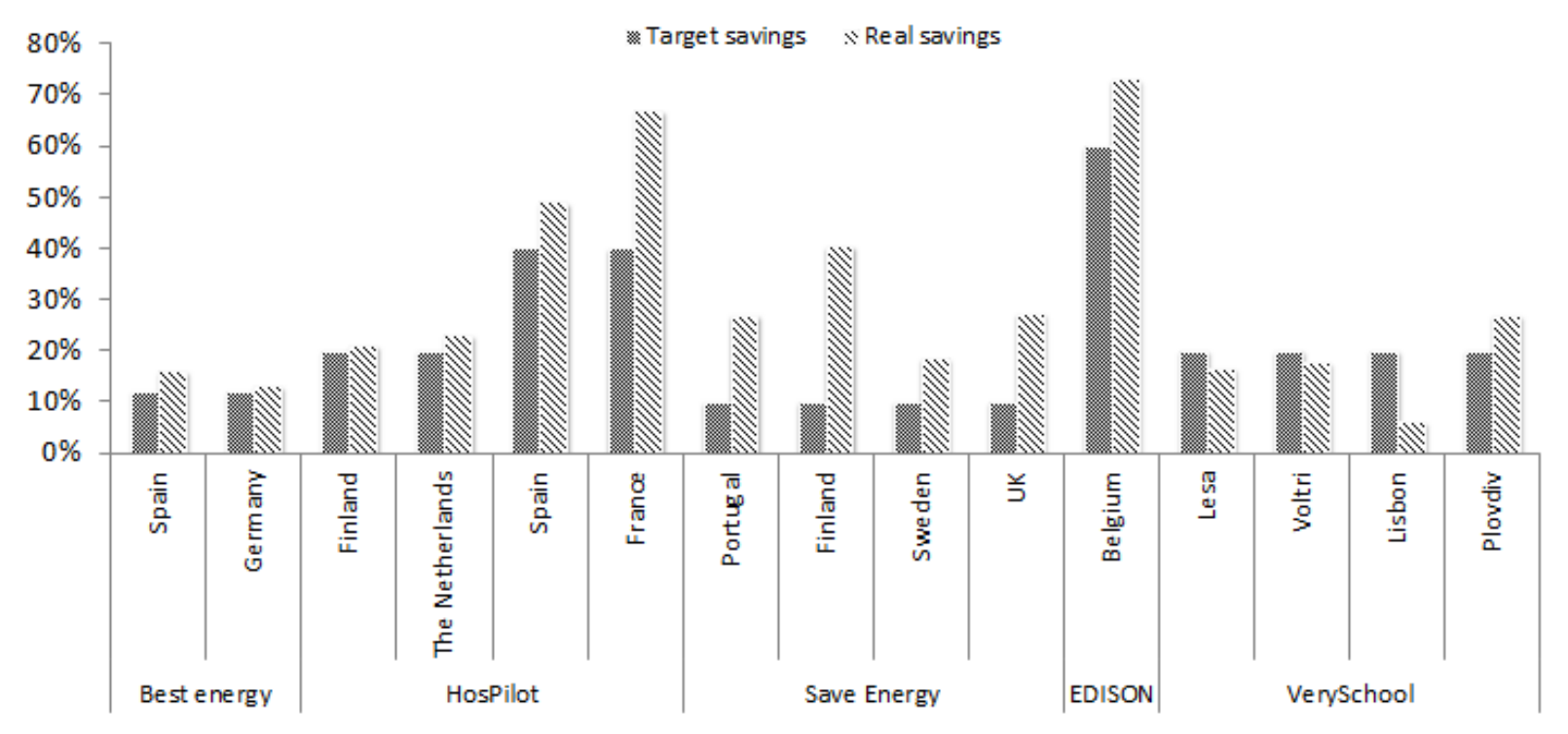

Figure 8. Total percentage of energy savings achieved within the different public building pilots. 
Table 1. EU projects analysed

\begin{tabular}{|c|c|c|c|}
\hline \multicolumn{2}{|c|}{ Social housing } & \multicolumn{2}{|c|}{ Public buildings } \\
\hline Project & Website & Project & Website \\
\hline 3e-Houses & www.3ehouses.eu & Best energy & www.bestenergyproject.eu \\
\hline E3SoHo & www.e3soho.eu & HosPilot & www.hospilot.eu \\
\hline eSESH & www.esesh.eu & Save Energy & www.ict4saveenergy.eu \\
\hline BECA & www.beca-project.eu & EDISON & www.project-edison.eu \\
\hline ICE-WISH & www.ice-wish.eu & GREEN@Hospital & www.greenhospital-project.eu \\
\hline \multirow[t]{7}{*}{ SHOWE-IT } & www.showe-it.eu & SMART Build & www.smartbuild.eu \\
\hline & & Smart CAMPUS & www.greensmartcampus.eu \\
\hline & & Smartspaces & www.smartspaces.eu \\
\hline & & VERYSchool & www.veryschool.eu \\
\hline & & EPLACE & www.eplaceproject.eu \\
\hline & & TEDS4BEE & www.teds4bee.eu \\
\hline & & SUNSHINE & www.sunshineproject.eu \\
\hline
\end{tabular}


Table 2. Sensors: measures and actions

\begin{tabular}{lr}
\hline Sensors & Use, collected data, operation \\
\hline Temperature & Provides a comfort indicator for each room of the house or building. \\
& Allows adjusting the indoor conditions based on outdoor conditions. \\
& Allows the user to transfer the energy excess from one room to another. \\
\hline Humidity & Gives data on comfort and indoor air quality. \\
\hline Lighting & Control of aperture depending on external lighting conditions. \\
\hline Occupancy & Regulation of temperature, lighting and ventilation depending on room occupancy. \\
\hline Opening & Heating and ventilation can be switched off in a room open to the outside. \\
\hline CO ${ }_{2}$ & Control of ventilation depending on indoor air quality. \\
\hline Consumption & Allows analysing the consumption of each resource (water, gas, electricity). \\
\hline
\end{tabular}


Table 3. Building Management Systems Skills

- Increase level of comfort.

- Individual tenant billing for services facilities.

- Remote monitoring and control of the systems in the building (such as Air Handling Units (AHUs), fire pumps, water pumps, electrical supply, Sewage Treatment Plan (STP), Water Treatment Plan (WTP), etc.).

- Improve maintenance enabling corrective and predictive actions.

- Monitor and optimise energy efficiency.
- Economizer control.

- Remote shut down.

- Equipment cycling.

- Scheduled-based heating, ventilation and cooling control (HVAC).

- Customizable \& comprehensive energy usage reports.

- Electrical demand limiting.

- Outside air reduction.

- Automated equipment scheduling.

- Leakage or left on limitations.

- Occupancy-based environmental controls.

- Optimal start/stop. 
Table 4. Number of Social housing and Public buildings projects.

\begin{tabular}{cccc}
\hline \multicolumn{2}{c}{ Social housing projects } & \multicolumn{2}{c}{ Public buildings projects } \\
\hline Number of buildings & 364 & Number of buildings & 230 \\
Number of dwellings & 7,626 & & \\
Users (tenants) & 11,218 & Users (users + visitors) & 770,993 \\
Total surface $\left(\mathbf{m}^{2}\right)$ & $\mathbf{4 7 9 , 9 5 5}$ & Total surface $\left(\mathbf{m}^{2}\right)$ & $\mathbf{5 , 5 4 6 , 3 1 8}$ \\
\hline
\end{tabular}


Table 5. Social Housing: potential annual savings at European level. Annual savings per dwelling Total annual savings

\begin{tabular}{ccc}
\hline Electric & $200 \mathrm{kWh} /$ dwelling & $4,471 \mathrm{GWh}$ \\
Thermal & $517 \mathrm{kWh} /$ dwelling & $11,559 \mathrm{GWh}$ \\
$\mathrm{CO}_{2}$ emissions & $92 \mathrm{~kg} \mathrm{CO} /$ dwelling & $2,056,843$ tons $\mathrm{CO}_{2}$ \\
Economic & $46 € /$ dwelling & $1,028 \mathrm{M} €$ \\
\hline
\end{tabular}


Table 6. Public buildings: potential annual saving at European level.

\begin{tabular}{ccc}
\hline & Annual savings per $\mathrm{m}^{2}$ & Total annual savings \\
\hline Electric & $6.58 \mathrm{kWh} / \mathrm{m}^{2}$ & $7,994 \mathrm{GWh}$ \\
Thermal & $5 \mathrm{kWh} / \mathrm{m}^{2}$ & $6,075 \mathrm{GWh}$ \\
$\mathrm{CO}_{2}$ emissions & $9.18 \mathrm{~kg} \mathrm{CO} / \mathrm{m}^{2}$ & $11,152,782$ tons $\mathrm{CO}_{2}$ \\
Economic & $5.3 € / \mathrm{m}^{2}$ & $6,054 \mathrm{M} €$ \\
\hline
\end{tabular}


Table 7. Main steps of a replication plan.

\begin{tabular}{cc}
\hline Steps & Goals \\
\hline 1 & Definition of the objectives \\
2 & Definition of the boundary conditions and project limitations \\
3 & Building energy audit \\
4 & Selection of the ICT system \\
5 & ICT implementation \\
6 & User engagement \\
7 & Analysis and optimization of building performance \\
8 & Measurement and verification of energy savings \\
9 & Dissemination of results \\
\hline
\end{tabular}

